\title{
Aprender en la universidad \\ análisis de aspectos que lo condicionan desde la perspectiva de profesores y alumnos de la Universidad Nacional de Cuyo (UNCuyo/Argentina) y de la Universidad Federal do Río de Janeiro (UFRJ/Brasil)
}

\author{
IDA LUCÍA MORCHIO \\ Universidad Nacional de Cuyo, \\ Mendoza, Argentina \\ ADRIANA MABEL FRESQUET \\ Universidade Federal do Rio de \\ Janeiro, Rio de Janeiro, RJ, Brasil
}

\section{RESUMEN}

Este trabajo es fruto de cuatro años de investigación. Durante 2007-2009, desarrollamos el proyecto "Aprender en la universidad. Representaciones, procesos, estrategias y factores implicados". La primera cohorte estaba integrada por alumnos y profesores de Ciencias de la Educación, Ciencia Política, Medicina y Trabajo Social, carreras que se cursan en la UNCuyo (Universidad Nacional de Cuyo) y de Pedagogía en la UFRJ (Universidad Federal de Río de Janeiro). En el proyecto Aprender en la universidad. Representaciones, procesos, estrategias y factores implicados (2009-2011) avanzamos con una segunda cohorte en la que consideramos las mismas instituciones, carreras y aspectos bajo análisis a fin de enriquecer el tratamiento de las variables y de extraer conclusiones sobre una base más amplia, sin perder la pertinencia y aplicabilidad de las mismas. Los resultados permiten mantener dos de las hipótesis centrales: que hay diferencias en la conceptualización de aprender que sostienen estudiantes y profesores y que las diferencias principales entre estudiantes $\mathrm{con} / \mathrm{sin}$ dificultad para aprender radican en los procesos y estrategias que emplean.

aprender; universidad; representaciones; estrategias; procesos; factores personales y contextuales. 
LEARNING AT COLLEGE AND UNIVERSITY: AN ANALYSIS OF CONDITIONING FACTORS FROM THE PERSPECTIVE OF PROFESSORS AND STUDENTS FROM UNIVERSIDAD NACIONAL DE CUYO (UNCUYO/ARGENTINA) AND UNIVERSIDAD FEDERAL DO RIO DE JANEIRO (UFRJ/BRASIL)

\section{ABSTRACT}

This paper is the result of four years of research. During 2007-2009, we developed the Project Learning at College and University. Representations, Processes, Strategies and Factors Involved. The first cohort was made up of students and professors from the Educational Sciences, Political Sciences and Social Work courses of studies, which are taught at UNCuyo (Universidad Nacional de Cuyo), and from the Pedagogy course of study, taught at the UFRJ (Universidad Federal de Río de Janeiro). In the Project Learning at College and University. Representations, Processes, Strategies and Factors Involved (2009-2011), we moved forward with a second cohort and analyzed the same institutions, courses of studies and aspects, in order to enrich the treatment of variables and draw conclusions on a more solid basis, keeping at the same time their pertinence and applicability. The results allow keeping two of the central hypotheses: that there are differences in the learning conceptualization of students and professors, and that the main differences between students with/without learning difficulties are in the processes and strategies they use.

KEYWORDS

learning; university; representations; strategies; processes; personal and context factors.

\section{APRENDER NA UNIVERSIDADE: ANÁLISE DE ASPECTOS QUE CONDICIONAM A PERSPECTIVA DE PROFESSORES E ALUNOS DA UNIVERSIDADE NACIONAL DE CUYO (UNCUYO/ARGENTINA) E DA UNIVERSIDADE FEDERAL DO RIO DE JANEIRO (UFRJ/BRASIL)}

\section{RESUMO}

Este trabalho é fruto de quatro anos de pesquisa. Durante o período de 2007-2009, desenvolvemos o projeto "Aprender na universidade. Representações, processos, estratégias e fatores implicados". A primeira etapa estava integrada por alunos e professores de ciências da educação, ciência política, medicina e trabalho social, cursos da Universidade Nacional de Cuyo (UNCuyo) y de pedagogia na Universidade Federal do Rio de Janeiro (UFRJ). Com esse mesmo projeto, já no período 2009-2011, avançamos em uma segunda etapa na qual consideramos as mesmas instituições, cursos e aspectos analisados, com o objetivo de enriquecer o tratamento das variáveis e extrair conclusões sobre uma base mais ampla, sem perder sua pertinência e aplicabilidade. Os resultados permitem manter duas das hipóteses centrais: 1) existem diferenças na conceituação de aprender pelos estudantes e pelos professores; e 2) as diferenças principais entre estudantes com/ sem dificuldade para apreender radicam nos processos e nas estratégias que utilizam.

PALAVRAS-CHAVE

aprender; universidade; representações; estratégias; processos; fatores pessoais e contextuais. 
A menudo se subestiman los beneficios educativos de las actividades asociadas a la investigación, en parte debido a que los vinculos entre la enseñanza y la investigación no son tan claros o tangibles. Es importante que la investigación en las instituciones de educación superior se emprenda no solamente por motivos de prestigio académico o de consideraciones económicas, sino también como parte de la renovación total y del desarrollo del aprendizaje, la enseñanza y las actividades de servicio público, incluyendo la difusión del conocimiento.

UNESCO, 1995, ap. 83, p. 33

\section{APRENDER EN LA UNIVERSIDAD HOY}

Sabemos que la misión de la universidad gira en torno de la docencia, la investigación y la extensión. Centrando la atención en la primera, la enseñanza adquiere sentido en función del aprendizaje que promueve. Sin embargo la universidad mantiene en términos generales un sistema en el cual no se realiza un seguimiento constante del aprendizaje. Entre la clase y el examen queda un espacio en el cual el alumno aprende. Se da por supuesto que todo alumno que ingresa "sabe" aprender y que todo profesor "sabe" cómo aprenden sus alumnos. En este sentido dice Torre Puente (1997, p. 16) "Con demasiada frecuencia los profesores suponemos que los estudiantes, por serlo, ya saben estudiar y, de no saberlo, creemos que no es competencia de los profesores invertir energía y tiempo en ello".

Por otra parte, calidad y excelencia son objetivos muy preciados en educación superior; calidad, en referencia al logro de resultados según un diseño previamente establecido y excelencia como "lo que se ha de hacer para alcanzar la optimización de lo posible" (Álvarez Pérez, 2002, p. 18). Entre los parámetros convencionales para estimarlos se cuentan, por ejemplo, los índices de retención, el desgranamiento, la tasa de deserción, la proporción entre ingresantes y egresados y la duración real de las carreras, pero pocas veces se incluyen indicadores de aprendizaje y, cuando se hace, los datos se refieren a resultados, no a procesos.

Se genera entonces el riesgo de entropía, entendida como pérdida de información de un sistema. En el plano local, la evaluación institucional de la Universidad Nacional de Cuyo (UNCuyo) advierte que "La entropía acecha siempre, tanto desde lo social y cultural en general, como desde la propia institución o desde las características individuales de cada participante" (UNCuyo, 1995, ap. 2.7, p. 32).

Tellez (1997) opina que tanto en círculos universitarios como extrauniversitarios suele circular la opinión de que la universidad está "superdiagnosticada", pero esto es así sólo cuando se entiende el diagnóstico como identificación y análisis de variables como financiamiento, productividad, descentralización administrativa, gratuidad, investigación, matrícula estudiantil, docencia, entre otros. No obstante quedan aspectos relevantes a los que no se les presta atención. 
Como integrantes de la comunidad educativa universitaria percibimos que la preocupación por hacer efectiva la acción de la universidad suele concretarse en la revisión y ajuste de planes de estudio, de servicios, de normativa, etc. Sin embargo, creemos que avanzar hacia una universidad que optimiza lo posible, reclama recuperar también las percepciones de alumnos y profesores en tanto protagonistas del hecho educativo con la expectativa de contribuir a la excelencia y a la calidad en términos de preparación profesional y desarrollo personal de los universitarios.

A la luz de estas consideraciones resulta pertinente y relevante indagar sobre el aprender, en tanto motor del desarrollo personal y -si se nos permite la expresión-, "corazón" de la vida en la universidad, puesto que a él se encaminan gran parte de los esfuerzos que invierte una comunidad educativa. Por otra parte, un aprendizaje efectivo repercute en el contexto social bajo la forma de un servicio profesional competente. Constituirlo en objeto de análisis implica aprovechar constructivamente una información que contribuye al funcionamiento de la universidad como un sistema abierto, flexible, dinámico.

El propósito que orienta la línea de investigación aprender en la universidad es llegar a una descripción en la que se identifiquen elementos que suelen pasar desapercibidos y que impactan sobre la efectividad del aprendizaje como base para diseñar acciones que promuevan en el alumno la toma de conciencia y el control de los procesos que realiza y en el profesor una revisión de sus prácticas docentes desde la perspectiva de quien aprende. En otras palabras, aprovechar constructivamente la información que brindan los actores -fuentes primarias- respecto de los condicionantes que actúan como factores facilitadores u obstaculizadores del desempeño.

En el proyecto bienal 2007-2009,"Aprender en la universidad. Representaciones, procesos, estrategias y factores implicados", ${ }^{1}$ se trabajó con una primera cohorte integrada por alumnos y profesores de cuatro carreras que se cursan en la UNCuyo y de una que se cursa en la Universidad Federal de Río de Janeiro (UFRJ). Al procesar la información -por grupos (estudiantes/profesores), por segmentos (alumnos que aprenden fácilmente/que tienen alguna dificultad para hacerlo), por carrera y por universidad- se abrió un gran espectro, lo cual derivó en que las elaboraciones para algunas categorías se apoyaran en pocos casos. Por esa razón se decidió tomar los resultados obtenidos hasta entonces como una primera etapa y ponerlos a prueba en nuevas muestras extraídas de las mismas carreras y

1 Evaluado y aprobado por la Secretaría de Ciencia, Técnica y Posgrado de la UNCuyo, Ministerio de Educación, Argentina, y registrado con el código 06-G443. El equipo de investigación está integrado por: doctora Ida Lucía Morchio (dir.); doctora Adriana Mabel Fresquet; especialista Gabriela Inés González; contadora María del Carmen Carrió; magister Lidia Diblasi; especialista Iris Noelia Maya; licenciada Mariela Lourdes González; licenciada Griselda Beatriz García; licenciada Guillermo Gallardo y licenciada María de Belén Pulvirenti. 
unidades académicas a fin de enriquecer el tratamiento de las variables en sí y de elaborar las conclusiones sobre una base más amplia, sin perder la pertinencia y aplicabilidad de las mismas.

Actualmente, en el proyecto bienal 2009-2011,"Factores personales y contextuales que se conjugan en el aprender en la universidad", ${ }^{2}$ se avanza con una segunda cohorte en la que se consideran las mismas instituciones, carreras y aspectos bajo análisis, el instrumento para recoger la información y los procedimientos para su tratamiento. Replicar el estudio en varias muestras se tomó como estrategia para poner a prueba los resultados obtenidos con muestras pequeñas (Ambrosi, 2008).

Sobre postulados de corte cognitivista se conceptualiza el aprender como un proceso interno y complejo que promueve el desarrollo personal y social y que puede ser autorregulado y mediado a través de la interacción social.

En el plano empírico, se analiza la información que brindan estudiantes y profesores, respecto de un mismo fenómeno: el aprender, examinando convergencias y divergencias entre las representaciones respectivas y manifestaciones -o no- de características que distinguen a alumnos que aprenden fácilmente de alumnos que aprenden con alguna dificultad (desde la autopercepción).

Las hipótesis centrales son: que estudiantes y profesores sostienen diferentes concepciones de "aprender" y atribuyen logros y dificultades a distintos motivos; que las diferencias entre estudiantes con/sin dificultad para aprender se ligan principalmente con los procesos y estrategias que emplean; que no existen diferencias esenciales entre los procesos cognitivos que realizan los alumnos de las carreras que ingresan al análisis (lo cual implica alumnos argentinos y brasileros). ${ }^{3}$

\section{CUATRO SITUACIONES PARA PROBLEMATIZAR EL APRENDIZAJE EN LA UNIVERSIDAD}

El planteo inicial remite a cuatro situaciones diferentes pero vinculadas entre sí. La primera -que habíamos anticipado- es que en la universidad se monitorea la calidad educativa en función de aprobados/desaprobados; promovidos/no promovidos; ingreso/desgranamiento. Sin embargo en un análisis de este tipo -cuyo valor no se discute- el alumno ingresa como una categoría abstracta y el aprendizaje rara vez está presente. Por otra parte, la excelencia como aspiración y como meta

2 Evaluado y aprobado por la Secretaría de Ciencia, Técnica y Posgrado de la UNCuyo, Ministerio de Educación, Argentina y registrado con el código 06-G524. El equipo de investigación está integrado por: doctora Ida Lucía Morchio (dir.); doctora Adriana Mabel Fresquet; especialista Gabriela Inés González; contadora María del Carmen Carrió; magister Lidia Concepción Diblasi; especialista Iris Noelia Maya; doctora Viviana Ruth Garzuzi; licenciada Mariela Lourdes González, licenciada Griselda Beatriz García.

3 También se exploran atribuciones causales y características de personalidad, aspectos que no se describen en este artículo por una cuestión de extensión. 
es un concepto siempre presente en el discurso, pero pocas veces se operativiza de un modo que permita contrastar en el plano empírico de qué modo la universidad optimiza lo posible. En definitiva, indagar sobre el proceso mismo de aprender abre una perspectiva diferente para pensar la excelencia y la calidad educativa.

La segunda situación es el riesgo de que estudiantes y profesores empleen la palabra aprender con ciertas diferencias de conceptualización ligadas al rol. Bruner (2001, p. 288) advierte: "se cree ordinariamente que la realidad se presenta de un modo idéntico para todos los hombres mentalmente sanos", sin embargo "ocurre a veces que usamos las mismas palabras pero no poseemos los mismos conceptos" (idem, p. 264). Esta cuestión merece atención porque no se trata de ideas aisladas, sino de verdaderas teorías implícitas que subyacen a las conductas y actitudes de los individuos.

La tercera situación es la trascendencia que tienen las experiencias de fracaso en el ámbito académico. Acordamos con Polaino-Lorente (1993) que fracasar en los exámenes disminuye la confianza en las propias capacidades e impacta en el nivel de aspiraciones, a la vez que contribuye a la desmotivación, genera una autopercepción de indefensión y hasta de culpabilidad ante los demás.

La cuarta son los condicionantes derivados de la naturaleza misma del aprender. Quien aprende, si bien es el protagonista de los procesos cognitivos, no siempre vuelve sobre ellos para capitalizar experiencias -hacer reajustes, reestructurar estrategias y desarrollar otras nuevas-, quizás porque desconoce la potencialidad autorregulatoria y autopoiética (Mayor; Suengas; González Márquez, 1993) que conlleva la metacognición.

Referido a quien enseña, ya dijimos que su actividad toma sentido en función de la existencia de otro que aprende, pero sucede que por tratarse de un proceso interno sólo se puede conocer y comprender en forma indirecta, a través de sus manifestaciones en la conducta o la verbalización de quienes lo protagonizan. Cabe entonces preguntarse ¿qué tiene presente el profesor cuando diseña las prácticas pedagógicas? Según Bandura (1982) la enseñanza responde generalmente a las representaciones previas del profesor respecto de lo que debe hacer y saber el alumno -supuestos que con frecuencia remiten a las experiencias vividas por quienes enseñan cuando estuvieron en situación de aprendices-. Rara vez ingresan a sus deliberaciones datos de base empírica o al menos anticipaciones de sentido sobre los procesos que deberá poner en juego el estudiante para aprender el contenido previsto, las dificultades que se le pueden presentar, las claves para anticiparlas preventivamente. En definitiva, profundizar en la comprensión de cómo aprenden los alumnos, conocer "los ecos" del proceso interno a través de la información que ellos mismos proveen, representa un insumo para repensar la enseñanza como instancia de mediación y evitar que se desconecte del aprender.

Si la universidad quiere promover estudiantes estratégicos a través de intervenciones de sentido orientador y preventivo, es importante que comprenda el aprender y para ello es inevitable que lo constituya en tema de estudio. 


\section{Y ENTONCES, ¿QUÉ SIGNIFICA APRENDER?}

El término aprendizaje es rico en matices ya que puede ser entendido como: "producto", es decir, el resultado de una experiencia o el cambio que acompaña a la práctica; como "proceso" en el que la atención se centra en el comportamiento; y como "función", en sentido de intencionalidad que guía la interacción con la información, los materiales, las actividades, las experiencias.

Encabezar el enunciado de la línea de investigación con el verbo aprender en lugar del sustantivo aprendizaje -por cierto más frecuente en la bibliografía y el discurso científico- no es casual, sino que anticipa el propósito de estudiar el fenómeno en su dinámica y desde la mirada de sus protagonistas; es decir, en tanto proceso y función.

El aprendizaje ha sido investigado desde aproximaciones diferentes. Algunos de los más conocidos son: el de las operaciones cognitivas que realiza quien aprende, accesibles a través de procedimientos experimentales; el de los resultados, sobre la base del análisis de calificaciones y estadísticas en variadas instancias de evaluación; el de las conceptualizaciones y experiencias de aprender estimadas desde el punto de vista de quienes participan en el proceso.

En el estudio que presentamos partimos de los interrogantes ¿Cuando se refieren a aprender, las representaciones de alumnos y de profesores coinciden o difieren? ¿Cómo proceden para aprender los alumnos de carreras diferentes? ¿Qué les ayuda? La experiencia indica que entre quienes dedican tiempo y esfuerzo al estudio, sólo algunos obtienen resultados satisfactorios. ¿A qué se debe? ¿En qué se diferencian quienes aprenden sin dificultad de quienes tienen algunas dificultades para lograrlo? ¿Se debe a los procesos y estrategias que emplean o a otro tipo de factores?

Se ha optado por preguntar directamente a profesores y a estudiantes su punto de vista sobre los aspectos analizados a fin de que los resultados admitan la transferencia al plano aplicado, es decir, próximo a la resolución de problemas. Respecto de la validez de estimar los procesos mentales, Monereo y sus colaboradores (1997, p. 100) ubican en un extremo a quienes defienden la absoluta inaccesibilidad a los procesos internos, "con el argumento de que no podemos utilizar los procesos mediante los que conocemos nuestra realidad circundante para conocer esos mismos procesos" y en el otro extremo a quienes aceptan el relato subjetivo y personal de lo que se piensa (introspección) como un método inmediato y directo para acceder a ellos. En consonancia con Monereo creemos viable este acceso cuando se informa sobre aprendizajes realizados en forma intencional y consciente.

\section{APRENDER A APRENDER}

Se entiende el aprender según los postulados de la psicología de la educación, en particular desde el enfoque de la psicología cognitiva. Bajo este paraguas se distingue una línea ligada al procesamiento de la información y la metáfora 
computacional que se centra principalmente en los procesos intelectuales -de mayor o menor complejidad, tales como percepción, atención, memoria, lenguaje, razonamiento- apelando a aproximaciones de corte experimental y una más amplia, orientada al estudio de los procesos mentales con el fin de comprender la conducta humana (Carretero, 2001). Nuestra mirada se orienta en esta segunda dirección y aborda aspectos como concentración, memoria; razonamiento, retención, recuperación, etc. en tanto elementos que se conjugan en un aprendizaje intencional realizado por un ser humano en interacción con un entorno.

La investigación sobre el aprendizaje desde supuestos cognitivistas se afianza a partir de la década de 1980 y entre los trabajos reconocidos por la comunidad científica se cuentan algunos que recuperan la propia experiencia del alumno que aprende y sobre esa base identifican diferencias entre quienes aprenden de forma superficial y quienes lo hacen de forma profunda; entre las estrategias para aprender que emplean los novatos y los expertos frente a un mismo contenido, etc. Se consideran como referentes clásicos en esta línea los desarrollos de Selmes (1988) y los de Novak y Gowin (1988).

Por su parte los investigadores social-cognitivos estudian la autorregulación, conceptualizada por Zimmerman, Kitsantas, Campillo (2005) como acciones, sentimientos y emociones autogenerados para alcanzar metas de aprendizaje. En la práctica la autorregulación consiste en un proceso autodirectivo mediante el cual los alumnos actúan de un modo proactivo respecto de sus procesos mentales.

También hace su aporte a nuestra conceptualización del aprender el enfoque de Mayor, Suengas, González Márquez (1993) sobre metacognición. Si bien son numerosos los autores que han tratado el tema y los artículos científicos publicados, recuperamos los postulados de Mayor por el sentido autoconstructivo que atribuye a la metacognición.

En la complementación entre dichas líneas se asume en esta investigación que quien aprende tiene la capacidad de volver con el pensamiento sobre sus procesos cognitivos, de actuar intencionalmente para controlarlos y de emplearlos estratégicamente. Con este supuesto se considera que el estudiante universitario puede -más aún, necesita- conocer y apropiarse de sus procesos cognitivos en un plano metacognitivo y por esta vía intervenir activamente en el desarrollo de la competencia para aprender. También se asume que, si bien es un proceso interno, puede ser mediado por otros.

Un indicador de la importancia que se le asigna al tema a partir del 2000 la divulgación de numerosos artículos y la publicación reciente de libros como $\mathrm{La}$ universidad ante la nueva cultura educativa. Enseñar y aprender para la autonomía (Monereo; Pozo, 2003); La enseñanza universitaria centrada en el aprendizaje (Prieto Navarro, 2008), Calidad del aprendizaje universitario (Biggs, 2008), El aprendizaje estratégico. Enseñar a aprender desde el currículo (Pozo; Monereo, 2000); entre otros. En ellos se aborda la problemática desde el ángulo del profesor universitario.

Otros desarrollos se aproximan a la cuestión desde el ángulo del estudiante, por ejemplo, Psicología del aprendizaje universitario: la formación en competencias 
(Pozo; Pérez Echeverría, 2009); Estrategias de aprendizaje para universitarios. Un enfoque constructivista (Amaya Guerra; Prado Maillard, 2007); Aprendizaje autorregulado. Enseñar a aprender en diferentes entornos educativos (Lanz, 2006).

Frente a este estado de situación, el aporte innovador del trabajo que hemos realizado es que el análisis se realiza a partir de la experiencia de los actores primarios y por otro lado que el destinatario no es sólo el profesor o el especialista, sino el mismo alumno universitario preocupado por aprender a aprender.

\section{ALGUNAS PRECISIONES SOBRE EL PROCESO DE INVESTIGACIÓN}

Es una investigación descriptiva, comparativa y explicativa, de tipo aplicado en la que se complementan miradas de corte cuantitativo y cualitativo.

El contexto de estudio es la universidad y el tema se trata desde la subjetividad de los actores con dos particularidades distintivas: el contraste entre concepciones y la incorporación de información de corte metacognitivo.

La población en estudio incluye muestras de treinta estudiantes y diez profesores seleccionadas en forma intencional de cuatro carreras de la UNCuyo: trabajo social y ciencia política ${ }^{4}$ y administración pública (que se cursan en la Facultad de Ciencias Políticas y Sociales), ciencias de la educación (que se cursa en la Facultad de Filosofía y Letras), medicina (que se cursa en la Facultad de Medicina) y de una de la UFRJ: pedagogía (que se cursa en la Facultad de Educación).

Se escogieron la UNCuyo y la UFRJ considerando, por un lado, que ambas son universidades públicas, dando ciertas características comunes a la población. Por otro, que profesoras de ambas instituciones forman parte del equipo de investigación, lo cual asegura el acceso a estudiantes y a profesores en sus ámbitos naturales y el control de factores contextuales durante la aplicación. Además, existe en la UNCuyo un programa de movilidad estudiantil que permite anticipar la posibilidad de financiamiento para que los investigadores viajen a países del Mercado Común del Sur (MERCOSUR) a fin de exponer en reuniones científicas los trabajos realizados.

Se trabajó con estudiantes de los dos primeros años previendo que en el bienio 2011-2013 se avanzaría en la misma línea de investigación con el siguiente objetivo: comparar el esquema de resultados obtenidos en alumnos de los dos primeros años (proyectos 2007-2009 y 2009-2011) con los resultantes de alumnos del tramo final de las carreras en estudio, a fin de estimar el desarrollo (o no) de competencias académicas en el transcurso de la carrera.

Los aspectos -o si se prefiere las variables, empleando el término en sentido amplio- que se analizan son: representaciones asociadas con el término aprender, autopercepción de competencia para aprender, indicadores de aprendizaje efectivo,

4 Se emplea el plural, ciencias políticas, cuando se hace referencia a la unidad académica y el singular ciencia política para referirse a la carrera de ciencia política y administración pública que se cursa en dicha unidad académica. 
elementos que favorecen y que obstaculizan el aprender en la universidad, procesos que realiza quien aprende (concentración, comprensión lectora, elaboración, recuperación, expresión), estrategias que emplea (planificar los tiempos, consultar y preguntar, tomar apuntes, ordenar el material, analizar, sintetizar, exponer, repasar) y factores que influyen (salud, trabajo, motivación, familia, relación con los compañeros, clima institucional).

Centrarse en estos aspectos no implica desconocer el impacto de las aptitudes, la preparación previa en el nivel medio, los factores contextuales institucionales y sociales, entre muchos otros, sin embargo se hace necesario un recorte para el estudio.

La información se recoge a través de una encuesta para profesores y una para alumnos y se procesa empleando una metodología cualitativa para las representaciones y cuantitativa para los aspectos restantes.

Estudiantes y profesores se tratan como grupos separados para contrastar sus percepciones respecto de qué significa aprender, qué conductas ponen de manifiesto un aprendizaje efectivo y qué factores facilitan u obstaculizan el proceso. A su vez, el grupo "estudiantes" se segmenta en función de la autopercepción de competencia personal para aprender en: alumnos que opinan que aprenden fácilmente y alumnos que opinan que tienen alguna dificultad para hacerlo. En cada segmento se analiza por carrera y por universidad el comportamiento de las variables: procesos, estrategias y factores.

\section{CONVERGENCIAS Y DIVERGENCIAS ENTRE ESTUDIANTES Y PROFESORES RESPECTO DEL APRENDER}

Desde una aproximación cualitativa, al analizar las connotaciones que adquiere el término aprender en profesores y en estudiantes por un lado y en estudiantes de diferentes carreras y universidades por otro, resulta que ciertos términos son los más frecuentes en ambos grupos y en todas las carreras, en especial: "adquirir conocimientos, saber, entender, conocer, comprender, interpretar, relacionar, internalizar".Junto con estos elementos, que pueden tomarse como comunes, se presentan algunas diferencias interesantes.

Llama la atención que los alumnos mencionan entre las palabras asociadas con aprender "equivocarse", "errar", lo cual no sucede en profesores. Es llamativo también que los alumnos reiteran más que los profesores términos que hacen referencia a la interacción, tales como "debatir", "discutir", "comunicación”, "cuestionar".

También se muestran algunas particularidades por carrera. Por ejemplo, en ciencias de la educación, ciencia política y administración pública y trabajo social se reitera la idea de conocer y saber, ligada con los procesos de pensamiento que se realizan para lograrlo, en cambio en medicina adquiere mayor presencia la práctica. Por su parte en pedagogía aprender se asocia no sólo con contextos de educación formal (escuela, universidad) sino también con ámbitos no formales como conversar, 
el cine o la televisión; recuperando expresiones textuales podría decirse que para aprender basta con "estar abierto a las experiencias". Conviene recordar que pedagogía representa en este estudio a alumnos y profesores de Brasil, mientras que el resto de las unidades de análisis son de Argentina.

Con respecto a los indicadores de aprendizaje efectivo, los alumnos ubican en primer término la capacidad de expresar con sus palabras lo aprendido, establecer relaciones entre los temas del programa y poder dar ejemplos, en cambio los profesores priorizan la resolución de situaciones problemáticas, la innovación y la transferencia a situaciones variadas.

Para la variable elementos que facilitan el aprender, la opción elegida con mayor frecuencia por los estudiantes es la explicación del profesor, seguida por la lectura de la bibliografía y en medicina, por las actividades propias de la carrera. Los profesores por su parte ponen en primer lugar la participación en clase, seguida por opciones que coinciden con las de los alumnos.

En cuanto a los elementos que dificultan el aprender, profesores y estudiantes coinciden en la mala previsión de los tiempos. Para los alumnos también influye la falta de esfuerzo suficiente, mientras que para los profesores tienen peso los problemas de comprensión lectora y la dificultad para relacionar contenidos con situaciones reales.

\section{¿CUÁLES SON LAS DIFERENCIAS MÁS NOTABLES ENTRE QUIENES APRENDEN FÁCILMENTE Y QUIENES APRENDEN CON DIFICULTAD?}

Desde una aproximación cuantitativa, al interior del grupo estudiantes y tomando como variable de corte la autopercepción de competencia para aprender se obtienen algunos resultados relevantes referidos a procesos, estrategias y factores.

Las diferencias más marcadas entre segmentos se manifiestan respecto de los procesos empleados para aprender. Para ejemplificar la situación presentamos los resultados que corresponden a algunos de los indicadores de Comprensión lectora y de Retención de lo estudiado. En ambos aspectos las diferencias entre segmentos se replican en todas las carreras.

Con el propósito de facilitar una mirada de conjunto se han sintetizado en un solo cuadro los datos por cohorte (primera y segunda), por carrera (ciencias de la educación, trabajo social, ciencia política, medicina y pedagogía), por segmento (aprende fácilmente, aprende con alguna dificultad) y por categoría de la variable (verdadero, a veces, falso). Por una cuestión de espacio, fue necesario apelar a algunas abreviaturas cuyo significado es: $\mathrm{F}$ (aprende fácilmente); $\mathrm{C} / \mathrm{D}$ (aprende con alguna dificultad); Verd. (verdadero); Av (a veces).

El Cuadro 1 permite observar el contraste entre segmentos frente al enunciado Tengo que leer varias veces para comprender, el cual es notable en dos carreras. Para trabajo social, según los datos de la primera cohorte, el ítem es verdadero para 
el $11,1 \%$ de quienes aprenden fácilmente y para el $75 \%$ de quienes lo hacen con dificultad. En la segunda cohorte se corrobora la diferencia entre segmentos, con porcentajes de $20 \%$ y $60 \%$ respectivamente. En ciencia política la tendencia tiene la misma dirección, siendo más alto el porcentaje de alumnos que tienen que leer varias veces para comprender entre quienes aprenden con dificultad, lo cual sucede en ambas cohortes, siendo mayores las diferencias en la segunda.

En otras carreras la distribución de la variable muestra diferentes comportamientos. Por ejemplo, en ciencias de la educación, en la segunda cohorte, señalan como verdadera esta afirmación el 13,6\% de quienes aprenden fácilmente, proporción que aumenta al $87 \%$ para los alumnos que consideran que aprenden con alguna dificultad. En cambio en la primera cohorte el porcentaje para ambos segmentos es semejante $(30,8 \%$ y $25,0 \%$ respectivamente) y además cambia la dirección de la tendencia. Algo análogo sucede en pedagogía pues en la segunda cohorte lo consideran verdadero el 7,7\% de quienes aprenden fácilmente y el 42,4\% de quienes lo hacen con dificultad; en cambio los porcentajes respectivos para la primera cohorte son $23,1 \%$ y $12,5 \%$.

En el Cuadro 2 se presentan los resultados correspondientes a otro de los indicadores de comprensión lectora. En este caso, en todas las carreras y en ambas cohortes es más alta la proporción de alumnos que aprenden con alguna dificultad que opinan que es verdadero para ellos el enunciado: Me cuesta comprender lo que leo. En cambio es más baja para quienes aprenden fácilmente.

En el Cuadro 3 se muestra el comportamiento de la variable procesos en el aspecto recuperación de lo aprendido. Si bien se observan frecuencias altas en la categoría a veces -lo cual resulta en cierto modo esperado, o si se prefiere razonable, por la complejidad del contenido a aprender-, hay un contraste significativo entre segmentos que mantienen la misma dirección en las cinco carreras, aunque con distinta intensidad. Esto significa que a los alumnos con alguna dificultad para aprender (autopercepción) les cuesta retener y recordar lo que estudian con más frecuencia que a quienes consideran que aprenden fácilmente. Sólo a modo ejemplificativo, en trabajo social las proporciones son muy semejantes en ambas cohortes y revelan que ninguno de los estudiantes que aprende fácilmente tiene dificultad para retener y recordar lo que estudia, en cambio esto le sucede casi a la mitad de quienes aprenden con alguna dificultad (44\% y $45 \%$ según cohorte).

$\mathrm{Al}$ analizar por segmentos la variable estrategias que emplea el estudiante no se observan diferencias significativas respecto de participación en clase, asistencia a horas de consulta, ordenar el material antes de empezar y tomar apuntes. Las diferencias se hacen más notables con respecto a analizar, sintetizar y buscar relaciones entre los temas del programa y el mayor contraste se muestra en las estrategias de revisión y repaso. Por ejemplo, si se observa en el Cuadro 4 el comportamiento de la segunda cohorte, el $80 \%$ de los alumnos de trabajo social que aprenden fácilmente dedica los últimos días antes de rendir al repaso, en cambio sólo lo hace el 44\% de quienes aprenden con alguna dificultad. También merece atención el contraste en medicina (60,0\% y $36,4 \%$ respectivamente) y en pedagogía ( $46,2 \%$ y $27,3 \%$ respectivamente). Aunque 
las proporciones son diferentes según carrera y cohorte, la tendencia entre segmentos se reitera, con la sola excepción de ciencias de la educación en la primera cohorte.

Referido a factores, en la población estudiada no se muestran diferencias significativas entre alumnos que aprenden fácilmente y alumnos que lo hacen con alguna dificultad respecto de salud, familia y motivación por la carrera. En cambio tener que atender obligaciones laborales simultáneamente al cursado de la carrera muestra diferencias que se reiteran en las dos cohortes. En la última columna del Cuadro 5 se puede observar que sólo para el 5,8\% y el 8,3\% de los alumnos que aprenden fácilmente (primero y segundo cohorte respectivamente) es verdadero que el trabajo no les deja tiempo para el estudio; en cambio lo es para el 20,7\% y el 17,6\% (primero y segundo cohorte respectivamente) de quienes consideran que aprenden con dificultad. En un análisis por carrera se advierte gran variabilidad en el comportamiento de este aspecto de la variable, lo cual condiciona las posibles generalizaciones. 

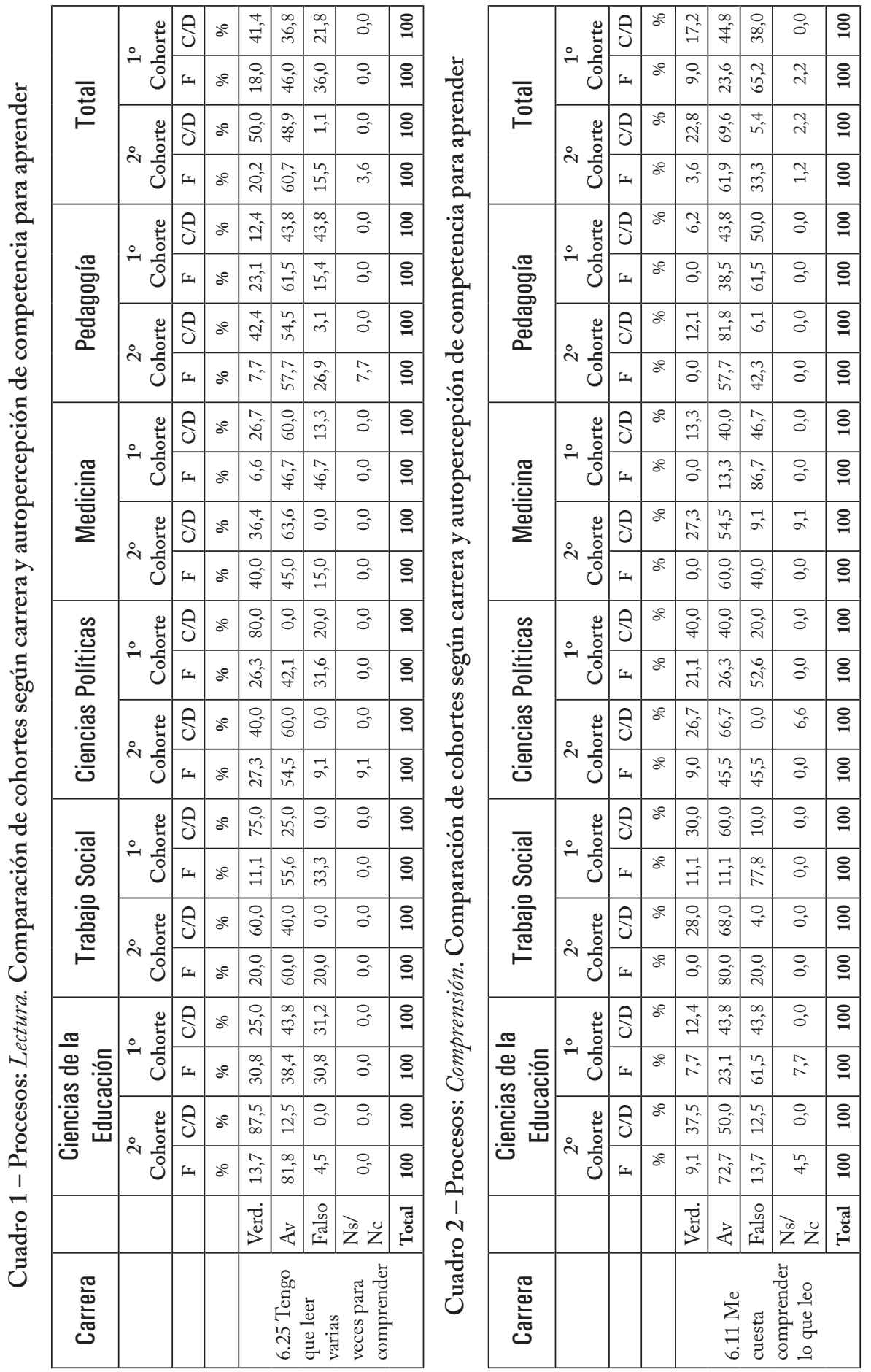


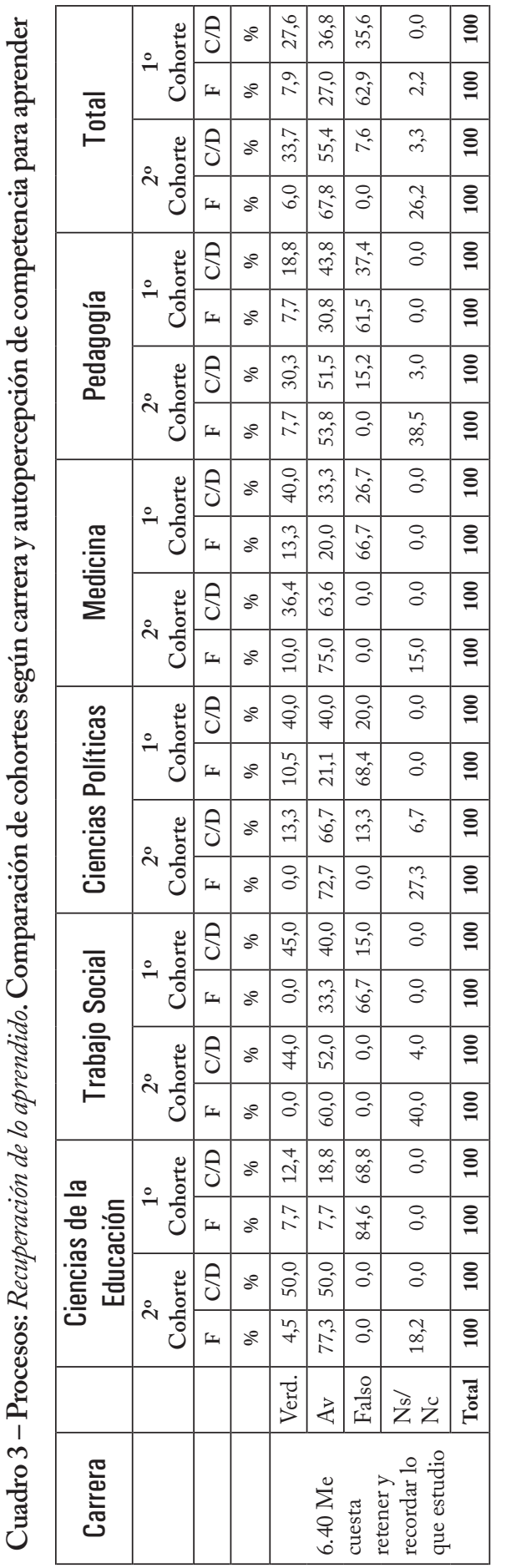

\begin{tabular}{|c|c|c|c|c|c|c|c|c|}
\hline \multirow{4}{*}{ 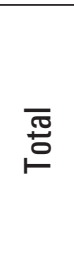 } & \multirow{2}{*}{ 둥 } & $\frac{8}{0}$ & de & $\begin{array}{l}\stackrel{0}{m} \\
\dot{m}\end{array}$ & $\begin{array}{l}\stackrel{0}{1} \\
\dot{m}\end{array}$ & $\dot{m}$ & : & 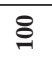 \\
\hline & & $I_{1}$ & $\partial^{\circ}$ & $\begin{array}{l}\dot{\sigma} \\
\stackrel{q}{q}\end{array}$ & $\begin{array}{l}\overrightarrow{0} \\
\hat{\sim}\end{array}$ & $\begin{array}{l}0 \\
\stackrel{N}{N}\end{array}$ & : & 8 \\
\hline & \multirow{2}{*}{$\therefore \frac{0}{0}$} & $\frac{\theta}{0}$ & $\partial^{\circ}$ & 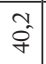 & $\hat{\text { in }}$ & 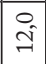 & $\vec{i}$ & 8 \\
\hline & & I & $a^{\circ}$ & $\begin{array}{l}3^{2} \\
\infty^{2} \\
i n\end{array}$ & $\begin{array}{l}0 \\
\vec{m}\end{array}$ & $\begin{array}{ll}12 \\
\alpha\end{array}$ & $\underset{-}{\Im}$ & $\stackrel{8}{ }$ \\
\hline \multirow{4}{*}{$\begin{array}{l}\text { 吾 } \\
\text { 兽 } \\
\text { 吾 }\end{array}$} & \multirow{2}{*}{$\stackrel{\frac{0}{0}}{\circ}$} & $\frac{\theta}{\delta}$ & $\partial^{\circ}$ & $\begin{array}{l}\vec{t} \\
\mathfrak{r}\end{array}$ & $\begin{array}{l}\infty \\
\tilde{y}^{-}\end{array}$ & $\begin{array}{l}\infty \\
\tilde{f} \\
\tilde{f}\end{array}$ & : & 으 \\
\hline & & I & $\partial^{\circ}$ & \begin{tabular}{|l|}
$\infty$ \\
0 \\
$\hat{n}$
\end{tabular} & $\begin{array}{l}+\dot{ } \\
\infty_{\infty}^{\prime}\end{array}$ & $\begin{array}{l}\infty \\
0 \\
\hat{\infty}\end{array}$ & : & 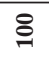 \\
\hline & \multirow{2}{*}{$\stackrel{\frac{0}{0}}{\frac{0}{0}}$} & ป & $\partial^{\circ}$ & 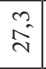 & $\stackrel{n}{i n}$ & $\stackrel{\vec{v}}{\vec{N}}$ & : & \& \\
\hline & & II & $\partial^{\circ}$ & $\begin{array}{l}n \\
\text { fo } \\
f\end{array}$ & $\begin{array}{l}\hat{\mathrm{s}} \\
\hat{\mathrm{N}}\end{array}$ & $\overrightarrow{\hat{v}}$ & $\stackrel{\infty}{\overbrace{}^{-}}$ & 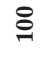 \\
\hline \multirow{4}{*}{ 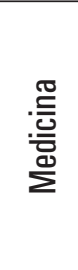 } & \multirow{2}{*}{$\circ \frac{0}{0}$} & i & $\partial^{\circ}$ & $\begin{array}{l}0 \\
\dot{i}\end{array}$ & $\begin{array}{l}0 \\
\hat{i}\end{array}$ & $\begin{array}{l}0 \\
8\end{array}$ & O. & 8 \\
\hline & & II & $\partial^{\circ}$ & $\hat{\mathrm{b}}$ & $\stackrel{\stackrel{m}{\vec{r}}}{\stackrel{2}{2}}$ & $\begin{array}{l}0 \\
\hat{i}\end{array}$ & O: & $\stackrel{8}{0}$ \\
\hline & \multirow{2}{*}{$\therefore \frac{\stackrel{0}{0}}{0}$} & i & $\circ^{\circ}$ & 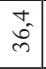 & $\begin{array}{l}\dot{t} \\
\dot{f} \\
q\end{array}$ & $\Rightarrow$ & $\Rightarrow$ & 8 \\
\hline & & 5 & $\therefore$ & $\begin{array}{l}0 \\
\dot{8}\end{array}$ & $\begin{array}{l}0 \\
\dot{0} \\
0\end{array}$ & $\begin{array}{l}0 \\
0\end{array}$ & : & 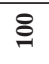 \\
\hline \multirow{4}{*}{ 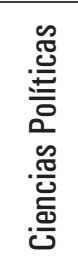 } & \multirow{2}{*}{$\circ \frac{0}{0}$} & i & $a^{\circ}$ & $\begin{array}{l}0 \\
i n \\
\text { in }\end{array}$ & $\begin{array}{l}0 \\
\dot{m} \\
\dot{m}\end{array}$ & $\begin{array}{l}0 \\
\text { in }\end{array}$ & O & 8 \\
\hline & & 5 & de & \begin{tabular}{|c|}
+ \\
$\infty$ \\
0 \\
0
\end{tabular} & $\overrightarrow{\vec{i}}$ & $\begin{array}{l}12 \\
\stackrel{2}{9} \\
-1\end{array}$ & $\because$ & 8 \\
\hline & \multirow{2}{*}{ ㄴ $\frac{\stackrel{0}{0}}{0}$} & O & $a^{\circ}$ & $\begin{array}{l}0 \\
8 \\
8\end{array}$ & $\hat{\tilde{s}}$ & $\begin{array}{l}m \\
\stackrel{2}{r}\end{array}$ & $\because$ & 8 \\
\hline & & II & $\therefore$ & है & $\begin{array}{l}t_{0} \\
\hat{\delta}\end{array}$ & O & : & 8 \\
\hline \multirow{4}{*}{ 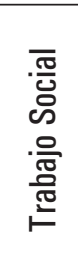 } & \multirow{2}{*}{$\stackrel{\frac{\pi}{0}}{\circ}$} & $\frac{8}{0}$ & $\partial^{\circ}$ & $\begin{array}{l} \\
\text { in } \\
\rightarrow\end{array}$ & in & $\begin{array}{l}0 \\
\text { हे }\end{array}$ & O & $\stackrel{7}{7}$ \\
\hline & & 5 & $\therefore$ & $\begin{array}{l}\dot{+} \\
\dot{f}\end{array}$ & $\dot{m}$ & ปี & : & $\stackrel{7}{7}$ \\
\hline & \multirow{2}{*}{ 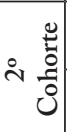 } & ปิ & $\partial^{\circ}$ & $\begin{array}{l}0 \\
\dot{f}\end{array}$ & $\begin{array}{l}0 \\
\stackrel{+}{+}\end{array}$ & $\stackrel{\circ}{\circ}$ & $\stackrel{\circ}{\circ}$ & 요 \\
\hline & & $I$ & $\therefore$ & $\begin{array}{l}0 \\
\dot{\infty} \\
\infty\end{array}$ & $\stackrel{0}{\stackrel{i}{0}}$ & O & : & 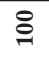 \\
\hline \multirow{5}{*}{ 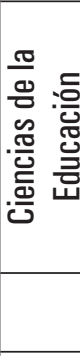 } & \multirow{2}{*}{$\therefore \frac{0}{0}$} & i & $a^{\circ}$ & $\begin{array}{l}\infty \\
\infty \\
0 \\
0\end{array}$ & $\begin{array}{l}\stackrel{\infty}{\infty} \\
\stackrel{\infty}{\sim}\end{array}$ & $\stackrel{+}{\mathfrak{I}}$ & : & $\stackrel{8}{\circ}$ \\
\hline & & I & $\partial^{\circ}$ & $\frac{n}{6}$ & $\overrightarrow{\hat{\imath}}$ & $\begin{array}{l}+ \\
20 \\
2\end{array}$ & : & 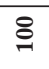 \\
\hline & \multirow{3}{*}{ 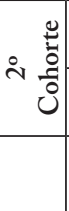 } & i & $a^{\circ}$ & $\begin{array}{l}0 \\
\dot{0} \\
i n\end{array}$ & : & O & : & \& \\
\hline & & 5 & $\partial^{\circ}$ & $\begin{array}{l}0 \\
\delta 0 \\
\end{array}$ & $\begin{array}{l}t_{0}^{\prime} \\
\text { है }\end{array}$ & : & : & 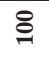 \\
\hline & & & & 㻤 & 齐 & 另 & हे zu & قี \\
\hline 苟 & & & & & & & & \\
\hline
\end{tabular}




\begin{tabular}{|c|c|c|c|c|c|c|c|c|}
\hline \multirow{4}{*}{$\begin{array}{l}\bar{\pi} \\
\text { 号 } \\
\end{array}$} & \multirow{2}{*}{ 은 } & i & $a^{\circ}$ & $\hat{\hat{i}}$ & $\begin{array}{l}n= \\
\Rightarrow\end{array}$ & $\begin{array}{l}\infty \\
\hat{0} \\
\hat{6}\end{array}$ & $\because$ & $\stackrel{8}{1}$ \\
\hline & & II & $\partial^{\circ}$ & $\begin{array}{l}\infty \\
i^{n}\end{array}$ & \begin{tabular}{|c|}
0 \\
$\hat{n}$ \\
\end{tabular} & $\frac{n}{\infty}$ & O) & 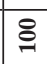 \\
\hline & \multirow{2}{*}{ 돈 } & s & $\partial^{\circ}$ & 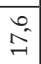 & $\begin{array}{l}\tilde{z} \\
\stackrel{d}{\sim}\end{array}$ & $\vec{n}$ & $\Rightarrow$ & $\stackrel{8}{2}$ \\
\hline & & II & $\partial^{\circ}$ & $\infty^{2}$ & \begin{tabular}{|l|}
12 \\
12 \\
7
\end{tabular} & $\begin{array}{l}0 \\
\text { in }\end{array}$ & $\approx$ & $\stackrel{ }{2}$ \\
\hline \multirow{4}{*}{ 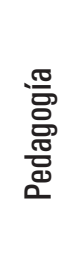 } & \multirow{2}{*}{ 운 $\frac{0}{0}$} & ঠ & $a^{\circ}$ & s. & $\begin{array}{c}\vec{n} \\
\stackrel{n}{n}\end{array}$ & $\hat{i n}$ & O. & 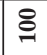 \\
\hline & & I & $\partial^{\circ}$ & 8 & $\stackrel{\therefore}{\therefore}$ & $\begin{array}{c}\hat{2} \\
\hat{\sigma}\end{array}$ & $\because$ & $\stackrel{8}{0}$ \\
\hline & \multirow{2}{*}{ 원 } & 8 & $\partial^{\circ}$ & $\stackrel{c}{\stackrel{2}{d}}$ & $\begin{array}{l}\dot{y} \\
\mathcal{g}\end{array}$ & ?े & $\vec{m}$ & 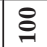 \\
\hline & & II & $\partial^{\circ}$ & $\Omega^{2}$ & $\tilde{z}$ & $\hat{\wedge}$ & ले & $\stackrel{8}{0}$ \\
\hline \multirow{4}{*}{ 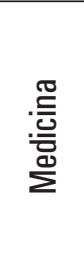 } & \multirow{2}{*}{ 은 } & 8 & $\partial^{\circ}$ & $\hat{\sigma}$ & $\hat{\sigma}$ & రం & O. & $\stackrel{8}{\circ}$ \\
\hline & & II & $\partial^{\circ}$ & $\therefore$ & $\begin{array}{l}0 \\
0\end{array}$ & $\therefore$ & O. & $\stackrel{8}{2}$ \\
\hline & \multirow{2}{*}{$\stackrel{\frac{\pi}{0}}{\frac{0}{0}}$} & 8 & $\partial^{\circ}$ & 8 & $O_{0}^{0}$ & $\begin{array}{l}0 \\
8\end{array}$ & $\stackrel{0}{0}$ & 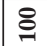 \\
\hline & & I & $a^{\circ}$ & in & $\begin{array}{l}0 \\
0\end{array}$ & î & O & $\stackrel{8}{0}$ \\
\hline \multirow{4}{*}{ 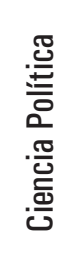 } & \multirow{2}{*}{ 은 } & 8 & $\sigma^{\circ}$ & $\begin{array}{l}0 \\
\text { in }\end{array}$ & \begin{tabular}{|l|}
0 \\
0 \\
\hdashline
\end{tabular} & $\begin{array}{l}\circ \\
\dot{q}\end{array}$ & $\because$ & $\stackrel{్}{ }$ \\
\hline & & II & $\partial^{\circ}$ & $\mathrm{n}^{2}$ & $\begin{array}{l}u_{2} \\
0 \\
-1\end{array}$ & $\begin{array}{l}f \\
\dot{f}\end{array}$ & ${ }_{0}^{0}$ & $\stackrel{8}{8}$ \\
\hline & \multirow{2}{*}{$\therefore \frac{0}{0}$} & 8 & do & $m^{2}$ & $\hat{s}$ & : & ${ }_{0}^{\circ}$ & $\stackrel{8}{\circ}$ \\
\hline & & I & $\partial^{\circ}$ & $\vec{\sigma}$ & of & $\hat{i}$ & O. & $\stackrel{8}{\circ}$ \\
\hline \multirow{4}{*}{ 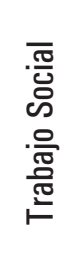 } & \multirow{2}{*}{ 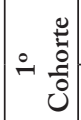 } & 8 & $\partial^{\circ}$ & : & $\begin{array}{l}0 \\
0 \\
0\end{array}$ & $\begin{array}{l}0 \\
\infty\end{array}$ & O. & $\stackrel{8}{\circ}$ \\
\hline & & II & $\partial^{\circ}$ & $\exists$ & $\Rightarrow$ & $\begin{array}{l}\infty \\
\stackrel{1}{\wedge}\end{array}$ & $O_{0}^{\circ}$ & $\stackrel{8}{2}$ \\
\hline & \multirow{2}{*}{$\stackrel{\frac{0}{0}}{\circ}$} & z & $\partial^{\circ}$ & $\begin{array}{l}0 \\
\dot{t}\end{array}$ & $\hat{\mathfrak{I}}$ & 앙 & : & $\stackrel{8}{\circ}$ \\
\hline & & I & $\partial^{\circ}$ & : & O. & is & $\because$ & $\stackrel{8}{0}$ \\
\hline \multirow{4}{*}{ 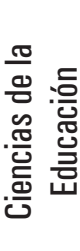 } & \multirow{2}{*}{$\therefore \frac{0}{0}$} & 8 & $\partial^{\circ}$ & $\begin{array}{l}\infty \\
\infty \\
\infty \\
-\infty\end{array}$ & $\begin{array}{c}\vec{f} \\
\tilde{I}\end{array}$ & $\begin{array}{l}\infty \\
\infty_{0}^{\infty}\end{array}$ & O. & $\stackrel{8}{\circ}$ \\
\hline & & I & $\sigma^{\circ}$ & $\begin{array}{l}+5 \\
n \\
n\end{array}$ & \begin{tabular}{|c|}
+ \\
$\infty$ \\
$\infty$
\end{tabular} & శ్ & $\stackrel{0}{\circ}$ & $\stackrel{8}{\circ}$ \\
\hline & \multirow{2}{*}{ ¿ $\frac{0}{0}$} & $\delta$ & $\partial^{\circ}$ & 8 & $\begin{array}{l}\tilde{L} \\
\tilde{\exists}\end{array}$ & $\begin{array}{l}12 \\
\hat{\infty}\end{array}$ & O. & $\stackrel{8}{\circ}$ \\
\hline & & I & $\partial^{\circ}$ & : & $\begin{array}{l}1 \\
\alpha^{\prime}\end{array}$ & $\begin{array}{l}\infty \\
\infty \\
\infty\end{array}$ & ${ }_{0}^{0}$ & $\stackrel{్}{ }$ \\
\hline & & & & Dं & & 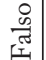 & $\overrightarrow{\bar{z}} \ddot{z}$ & हีّ \\
\hline 营 & & & & & 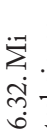 & & 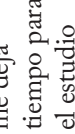 & \\
\hline
\end{tabular}




\section{ALGUNAS PROPUESTAS PARA TRANSFERIR LOS RESULTADOS DE LA INVESTIGACIÓN EN EL PLANO DE LA ACCIÓN}

Los resultados de la segunda cohorte corroboran la mayoría de los resultados de la primera y en ambas se mantiene la hipótesis de que los factores que explican principalmente la diferencia entre estudiantes universitarios con/sin dificultad para aprender son los procesos y estrategias que emplean.

Sobre la base de la aproximación cualitativa se advierten diferencias entre la conceptualización de aprender que tienen alumnos y profesores, como también entre lo que docentes y alumnos consideran indicadores de aprendizaje efectivo. Esta situación, adquiere relevancia si se piensa que las expectativas respectivas influyen a la hora de evaluar y de prepararse para una evaluación.

Asimismo se observan algunas diferencias entre la percepción de alumnos y profesores respecto de elementos que facilitan y factores que dificultan el aprender en la universidad, otra de nuestras hipótesis de trabajo.

También se puede mantener la hipótesis de que no existen diferencias esenciales en los procesos que emplean para aprender los alumnos de la UNCuyo y de la UFRJ.

Considerando que ésta es una investigación de modalidad aplicada -cuya finalidad es explicar un fenómeno en un contexto acotado, aportando criterios para la toma de decisiones y pautas para resolver un problema-, se ofrecen a continuación algunas reflexiones y propuestas a fin de tender un puente entre los resultados obtenidos y su transferencia al plano aplicado.

En este sentido, el enfoque del estudio realizado y el aporte a la discusión para la proyección al plano aplicado se aproximan al planteo de Pérez Serrano (1988, p. 51) quien advierte que

Todo esfuerzo de investigación en educación tiene como último propósito llegar a un conocimiento que pueda utilizarse para la acción, ya se trate de una acción a nivel político, ya de un cambio de la práctica en la clase.

Atendiendo a la diferencia entre lo que alumnos y profesores consideran indicadores de aprendizaje efectivo, se señala la importancia de que el profesor haga explícitas sus expectativas y genere instancias para ejercitar conductas afines con ellas. Cabe pensar que quizás el estudiante no piensa en innovaciones ni expresa puntos de vista divergentes porque no sabe que el profesor lo espera y lo valora. Algo semejante sucede con la transferencia de lo que se aprende a situaciones variadas.

Otra propuesta es estrechar el vínculo entre la enseñanza y el aprendizaje. Desde el marco teórico de esta investigación enseñar no es equivalente a "mostrar" lo que hay que aprender sino a actuar como mediador entre un contenido -que pueden ser conceptos, procedimientos, actitudes- $\mathrm{y}$ los procesos internos que es preciso realizar para aprenderlo. En la práctica se trata, por ejemplo, de compartir 
objetivos, de dialogar sobre las características y la funcionalidad del aprendizaje a lograr; de recuperar ideas previas que sirvan como anclaje de las ideas nuevas; de anticipar la secuencia a recorrer, las operaciones cognitivas implicadas, las estrategias para monitorear el propio desempeño, el sentido de la evaluación que acompaña el proceso y de la que se toma para la acreditación.

Volviendo sobre "equivocar" y "errar" que los alumnos mencionaron entre los términos asociados con el aprender, cabe pensar que los entienden como experiencias vividas en un camino no lineal hacia la apropiación, expresión y aplicación de un contenido.

Si se tiene presente que el aprendizaje es un proceso interno, tomar el error sólo como lo que hay que corregir o lo que determina qué calificación corresponde al alumno es desconocer una inestimable fuente de información. En cambio, si el alumno genera el hábito de analizarlos y aprender a partir de ellos y el profesor descubre en ellos pautas para reorientar la enseñanza (volviendo sobre aspectos que no quedaron claros, aportando pistas procedimentales que no se tuvieron en cuenta inicialmente, regulando ritmos, evaluando para sí mismo la eficacia de diferentes metodologías, etc.) se aprovecha su riqueza potencial.

Desde otra perspectiva, entender el error como un momento en el proceso de aprender lleva a revisar teorías implícitas y actitudes de alumnos y de profesores respecto de la evaluación. Aquí la propuesta es que la evaluación se constituya en una oportunidad de aprender y que si hubo dificultades, que el alumno comprenda en qué consistieron y se disponga a trabajar sobre ellas; y que el profesor asuma una actitud activa y favorable para analizar junto con él los errores, omisiones, etc., orientándolo sobre estrategias para superarlos y quizás ofreciéndole nuevas instancias para que ponga a prueba sus avances.

Si se adopta la perspectiva de la psicología humanística -cuyos postulados giran en torno al ser y el llegar a ser en sentido de autorrealización- se abre una aspiración más profunda: que el estudiante universitario se posicione frente a la carrera en términos de un proyecto propio, elegido por él, que se va construyendo a través de cada contenido que aprende, de cada experiencia que vive, y desde esta posición -en la que se complementan libertad y responsabilidad- "se evalúe" respecto de lo que necesita saber y saber hacer para ser un profesional competente.

En el campo de los procesos, un tema preocupante son las dificultades en la instancia de lectura, que representa generalmente la fase inicial del estudio y que condiciona las restantes.

La comprensión lectora -que mostró diferencias entre los estudiantes que consideran que aprenden fácilmente y los que se perciben con alguna dificultad para aprender- no es una cuestión de todo o nada, por ese motivo algunos ingresantes enfrentan en los primeros años dificultades para procesar diferentes géneros académicos. Una posible explicación de lo que sucede es que con frecuencia el material 
les resulta abstracto y complejo porque no fue escrito pensando en ellos como destinatarios. La bibliografía está integrada por fragmentos de la producción de la comunidad científica de una disciplina, que comparte un conjunto de conocimientos que suelen darse por supuestos. Frente a esta situación, Carlino (2006) plantea la necesidad de una "alfabetización académica", que se logra cuando los miembros de una comunidad disciplinar guían a los aprendices hacia sus modos particulares de comprender y producir textos.

Sólo algunos estudiantes ingresan con el equipamiento conceptual y procedimental apropiado para procesar contenidos con alto nivel de abstracción, de allí la importancia de comenzar haciendo juntos y avanzar en dirección a una autonomía progresiva, acompañando al alumno con información sobre su desempeño. A través de esta propuesta, en la que resuenan los ecos de los aportes referidos al modelado social (Bandura, 2004) o a la interacción en la "Zona de Desarrollo Próximo"(Vigotsky, 1999), lograr un aprendizaje efectivo se convierte en una meta compartida por quien enseña y quien aprende.

Si se acepta que enseñar cómo aprender forma parte del enseñar una materia y que la lectura y la escritura constituyen prácticas para la asimilación, producción y comunicación de un conocimiento que se vincula con la lógica de cada área científica, resulta fundamental su incorporación como contenido del curriculum.

En otro plano, al analizar los resultados para variables que se cruzan entre sí quedan a la vista algunas contradicciones. Por ejemplo, en el ítem referido a factores que obstaculizan el aprender la respuesta más frecuente es la mala previsión de los tiempos, mientras que en la variable estrategias, en las cinco carreras y en ambos segmentos predomina la tendencia a responder que es verdadero que planifica los tiempos. Frente a esta situación resultaría interesante promover procesos reflexivos y metacognitivos a través de los cuales el estudiante vuelva con el pensamiento sobre las experiencias vividas y aprenda a regular la propia conducta en función de factores favorecedores y factores obstaculizadores.

Los beneficiarios principales de esta investigación son los alumnos y los profesores. A la vez los resultados se transfieren a la universidad como aporte para revisar concepciones y acciones vinculadas con el aprender y a los servicios de orientación como insumos a tener en cuenta para el diseño de programas y acciones de apoyo al estudiante.

Recuperando algo dicho al comienzo, la motivación por recuperar la percepción de estudiantes y profesores se sustenta en la presunción de que puede aportar insumos de base para orientar al alumno respecto de aspectos que inciden en el logro de un "buen aprendizaje" y para invitar el profesor a diseñar estrategias pedagógicas pensando no sólo desde quien enseña sino también desde quien construye el aprendizaje. 
Por otra parte, es un modo de evitar la pérdida de información del sistema -entropía- capitalizando la experiencia de los protagonistas principales de la interacción educativa para revisar situaciones que generan el terreno propicio para el fracaso académico, la demora y hasta el abandono de los estudios.

Además, contribuir al desarrollo de la competencia para aprender y con ella la calidad educativa en términos de efectividad del aprendizaje representa una forma de aproximar la igualdad de oportunidades y la excelencia académica, que a veces se presentan distantes.

En síntesis, en el plano de la investigación los resultados manifiestan la necesidad de continuar con estudios que aporten insumos para una mejor comprensión del fenómeno y en proyección al plano aplicado se ingresa al debate sobre la importancia de que el aprender se constituya en un contenido de aprendizaje tan relevante como el contenido disciplinar en la preparación de un profesional competente para seguir aprendiendo a lo largo de la vida.

\section{REFERENCIAS}

Álvarez Pérez, Pedro. La función tutorial en la universidad. Una apuesta por la mejora de la calidad de la enseñanza. Madrid: EOS, 2002.

Amaya Guerra, Jesús; Prado Maillard, Evelyn. Estrategias de aprendizaje para universitarios. Un enfoque constructivista. México: Trillas, 2007.

Амвrosi, Hugo. La verdad de las estadísticas. Aprender con los datos. Buenos Aires: Lumiere, 2008.

Bandura, Albert. Teoría del aprendizaje social. Madrid: Espasa Calpe, 1982.

Ejercicio de la eficacia personal y colectiva en sociedades cambiantes. In: Bandura, Robert (Ed.). Auto-eficacia: cómo afrontamos los cambios de la sociedad actual. Bilbao: Desclée de Brouwer, 2004. p. 19-54.

Biggs, John. Calidad del aprendizaje universitario. Madrid: Narcea, 2008.

Bruner, Jerome Seymour. El proceso mental en el aprendizaje. Madrid: Narcea, 2001.

Carlino, Paula. Escribir, leer y aprender en la universidad. Una introducción a la alfabetización académica. Buenos Aires: Fondo de Cultura Económica, 2006.

Carretero, Mario. Introducción a la psicología cognitiva. Buenos Aires: Aique: 2001.

Lanz, María Zulma (Comp.). Aprendizaje autorregulado. Enseñar a aprender en diferentes entornos educativos. Buenos Aires: Novedades Educativas, 2006.

Mayor, Juan; Suengas, Aurora; GonZÁlez Márquez,Javier. Estrategias metacognitivas. Aprender a aprender y aprender a pensar. Madrid: Síntesis, 1993.

Monereo, Carles; Pozo, Juan Ignacio (Eds.). La universidad ante la nueva cultura educativa. Enseñar y aprender para la autonomía. Madrid: Síntesis, 2003. 
Monereo, Carles (Coord.); Castelló, Monserrat; Clariana,Mercè; Palma, Monserrat; Pérez Cabaní, María Lluïsa. Estrategias de enseñanza y aprendizaje. Formación del profesorado y aplicación en el aula. Barcelona: Graó, 1997.

Morchio, Ida Lucía (Dir.). Aprender en la universidad: representaciones, procesos, estrategias y factores implicados. Análisis desde la perspectiva del alumno y del profesor. Facultad de Filosofía y Letras. Proyecto Bienal 2007-2009. SeCTyP, UNCuyo, código 06-G443.

. (Dir.). Factores personales y contextuales que se conjugan en el aprender en la universidad. Análisis desde la percepción de sus protagonistas (UNCuyo y UFRJ) y desde archivos documentales (UNCuyo). Facultad de Filosofía y Letras. Proyecto Bienal 2009-2011. SeCTyP, UNCuyo, código 06-G524.

Novak, Joseph; Gowin, Bob (1988). Aprendiendo a aprender. Barcelona: Martínez Roca. Polaino-Lorente, Aquilino. Procesos afectivos y aprendizaje. In: Beltrán, Jesús. Intervención psicopedagógica. Madrid: Pirámide, 1993.

Pérez Serrano, Gloria. Investigación cualitativa. Tomo I. Madrid, 1988.

Pozo, Juan Ignacio; Monereo, Carles (Coords.). El aprendizaje estratégico. Enseñar a aprender desde el currículo. Madrid: Santillana, 2000.

Pozo, Juan Ignacio; Pérez Echeverría, María del Puy (Coords.). Psicología del aprendizaje universitario: la formación en competencias. Madrid: Morata, 2009.

Prieto Navarro, Leonor (Coord.). La enseñanza universitaria centrada en el aprendizaje. Barcelona: Octaedro, 2008.

Selmes, Ian. La mejora de las habilidades para el estudio. Barcelona: Paidós, 1988.

Unesco. Documento sobre politicas para el cambio y el desarrollo de la educación superior. Caracas, feb. 1995.

Tellez, Magaldy. Sobre el carácter de la crisis actual de la universidad. ¿Qué es lo que está en crisis? La universidad hoy, Universidad de Buenos Aires: Buenos Aires, año VI, 10,1997. Torre Puente, Juan Carlos. Aprender a pensar y pensar para aprender. Estrategias de aprendizaje. Madrid: Narcea, 1997.

Universidad Nacional de Cuyo - UNCuyo. Evaluación institucional de docencia universitaria. Mendoza, oct. 1995. Disponible en: <http://www.ediunc.uncu.edu.ar/ catalogo/ficha/18/Evaluacin-Institucional-de-la-Universidad-Nacional-de-Cuyo-IAutoevaluacin $>$. EDIUNC: 1996.

Vigotski, Liev Semionovich. El desarrollo de los procesos psicológicos superiores. México: Grijalbo, 1988.

Zimmerman, Barry; Kitsantas, Anastasia; Campillo, Magda. Evaluación de la autoeficacia regulatoria: una perspectiva social cognitiva. Evaluar, Universidad Nacional de Córdoba: Córdoba, n. 5, oct. 2005. 


\section{SOBRE LAS AUTORAS}

IDA Lucía MoRchio es doctora en educación por la Universidad Nacional de Cuyo (Argentina). Profesora de la misma institución.

E-mail: lucymorchio@speedy.com.ar

Adriana Mabel Fresquet es doctora en ciencias psicopedagógicas por la Pontificia Uniersidad Católica Argentina (Argentina). Profesora de la Universidade Federal do Rio de Janeiro (UFRJ).

E-mail: adrianafresquet@fe.ufrj.br

Recebido em maio de 2011

Aprovado em novembro de 2011 